\title{
Speed Response and Performance Degradation of High Temperature Gamma Irradiated Silicon PIN Photodiodes
}

\author{
${ }^{1}$ Abd El-Naser A. Mohamed, ${ }^{2}$ Nabil A. Ayad, ${ }^{3}$ Dr. Ahmed Nabih Zaki Rashed, ${ }^{4}$ Hazem M. El-Hageen \\ ${ }_{1,3,4}$ Dept. of Electronics and Electrical Communication Engineering, \\ Faculty Electronic Engineering, Menouf, Egypt \\ ${ }^{2,4}$ Atomic Energy Authority, Naser City, Cairo, Egypt
}

\begin{abstract}
In the present paper, we have been investigated deeply and parametrically the speed response of Si PIN photodiodes employed in high temperature-irradiated environment. The radiation-induced photodiodes defects can modify the initial doping concentrations, creating generation-recombination centres and introducing trapping of carriers. Additionally, rate of the lattice defects is thermally activated and reduces for increasing irradiation temperature as a result of annealing of the damage. Nonlinear relations are correlated to investigate the current-voltage and capacitance-voltage dependences of the Si PIN photodiodes, where thermal and gamma irradiation effects are considered over the practical ranges of interest. Both the ambient temperature and the irradiation dose possess sever effects on the electro-optical characteristics and consequently the photo-response time and SNR of Si PIN photodiodes. In this paper, we derive the transient response of a Si PIN photodiode for photogeneration currents, when it is exposed to gamma radiation at high temperature. An exact model is obtained, which may be used to optimize the responsivity and speed of these irradiated devices over wide range of the affecting parameters.
\end{abstract}

\section{Keywords}

Radiation effects, PIN photodiode, Optoelectronics, Transient current, Dark current, Photocurrent, Quantum efficiency, Responsitivity.

\section{Introduction}

Since several years, photonic and lightwave technology is seriously considered for optical access communication and monitoring applications in space borne local communication systems and nuclear projects. A major problem in these environments is the presence of radiation fields. Two types of damage affect the electronic devices when they are exposed to the radiation $[1,2]$. The first one is ionization damage, it is a transitory damage. In contrast, displacement damage is considered permanent. For several reasons, the interest of study of the effects of performance of devices in high-temperature electronics is developing rapidly. If these components are to be used in a radiation environment, knowledge about the degradation under high-temperature irradiation conditions is highly desirable.

The current-voltage technique is used to measure the rate of carrier creation and so the generation or the recombination rate $[3,4]$. On the other hand, the capacitance-voltage technique in reverse bias direction, is used to determine doping profiles of a semiconductor [4-6]. The capacitance measurements give information about fixed impurity states and defect centers in the band gap. Device testing, adequate system shielding and radiation tolerant design are some fundamental steps in the methodology or in the radiation hardness assurance [7, 8] that are needed to ensure the correct performance and efficiency of electronics during system life. But, there is an increasing interest in the development of accurate modeling and simulation techniques to predict device response under different radiation conditions.

In the present study, we have shown the modeling basics of a PIN photodiode device with the maximum possible precision, in order to predict the frequency response behaviour of Si PIN photodiodes when they were irradiated to different dose of high-temperature gamma radiation environments.

\section{Physical Modeling Basics}

Radiation damage produces defects which can result in three main effects on photodiode devices as following:

- The increase in dark current can be related to the minority carrier lifetime of the semiconductor if the generation-recombination is dominated by mid-band levels caused by defects. Another source affecting the dark current could be ionizing damage to the surface of the device.

- Degraded photocurrent as defects act as electron or hole trapping centers for the photogenerated pairs. The defects may be primary defects, i.e. defects which originate directly from atomic displacements, or secondary defects resulting from the interaction of mobile primary defects with impurities. Many defects will recombine leading to an immediate repair of the lattice. However, some will combine to form stable defects such as interstitials, di-vacancies, vacancy-impurity complexes, vacancy-dopant complexes, and larger clusters. These defects form effective recombination and trapping centers resulting in a decrease in the minority carrier lifetime, carrier density and carrier mobility. Defect centers position in the band gap determines their activity and hence the conduction mechanism in devices made from such material [9]. Deep traps are defects whose ionization energy, $\mathrm{E}$, is much greater than $\mathrm{kBT}(\mathrm{kB}$ is the Boltzmann constant and $T$ is the temperature). They trap free carriers with the consequence that they reduce the conductivity considerably. In contrast, shallow traps are easily ionized at equilibrium since $\Delta \mathrm{E}<<\mathrm{kBT}$, and so they increase the conductivity by releasing trapped carriers. In depleted regions they contribute to the space charge and the voltage required for full depletion. Generation-recombination (g-r) centers are situated near the centre of the band gap, in which position their trapping for electrons and for holes is comparable, and so they easily generate or recombine $\mathrm{e}-\mathrm{h}$ pairs. Then the free carriers are removed to reduce the conductivity. Defect centers can also act as compensation centers in the electrical neutral bulk of a semiconductor. Here, the deep levels are not easily ionized at equilibrium and have the effect of locking away free carriers to reduce the conductivity. The response degradation [8] is probably related to type inversion of the low-doped layer from $n$ to $p$-type. At low integrated fluence, the radiation forming acceptor state levels compensate the donor states until the effective doping concentration Neff is reduced to that of the intrinsic semiconductor. At higher fluences, the 
effective doping is mainly provided by the radiation induced defects. The concentration of majority carriers decreases with the irradiation fluence.

- Degraded rise and fall times due to de-trapping or a reduction in the carrier mobility [10]: The decrease in photocurrent and the increase in the dark current are expected to be the major changes in thin junction devices such as photodiodes. The change in the device response, rise and fall times are expected to be small, but still require measuring. Three main factors limit the speed of response of a photodiode. These are [11]:

a) The drift time of the carriers through the depletion region;

b) The diffusion time of the carriers generated outside the depletion region;

c) The time constant incurred by the capacitance of photodiode with its load and its associated circuit.

Photons that penetrate the semiconductor can be absorbed and its energy can be utilized in the generation of $\mathrm{e}-\mathrm{h}$ pairs. The model that describes the rate of generation is [12]:

$G_{\text {opt }}(x)=(1-r) \eta \frac{p_{0} \alpha}{h v} \exp (-\alpha x)$

where $r$ is the reflection coefficient, Previous reports in literature have stated that is independent of dose for $1 \mathrm{MeV}$ electron irradiations up to $5 \times 1015 \mathrm{~cm}-2$ [13]. $\eta$ is the quantum efficiency, $P_{0}$ is the incident light intensity, $h$ is the Planck constant, $v$ is the photon frequency, $\alpha$ is the absorption coefficient and $x$ is the depth variable. The optical spectral response of a PIN photodiode is called the optical sensitivity or the responsivity and it is related to the total photon-induced current. If the width of the $p$-layer is much thinner than $1 / \alpha$, the photoninduced current in the $p$-layer does not contribute to the total photocurrent.

The current-voltage and capacitance-voltage characteristics, in the dark and under illumination are highly sensitive to the radiation-induced change of the minority carrier lifetime T. In general, the damage coefficients for the mean minority carrier lifetime in semiconductors depend on the following parameters; type and energy of the incident particle, kind of material, resistivity, types and concentration of impurities, injection level, temperature and elapsed time after irradiation [14, 15]. Also a semiconductor $\mathrm{p}-\mathrm{n}$ junction acts as a capacitor. The depletion region capacitance of a uniformly doped lifetime diode at full depletion may be expressed in terms of the dielectric constants $\varepsilon_{0}, \varepsilon_{r}$ In this situation the effective carrier concentration is evaluated:

$$
N_{e f f}=\frac{2 C^{2}}{q \varepsilon_{r} \varepsilon_{0} A^{2}} V
$$

Where $A$ is the active diode area, $q$ is the electronic charge and $\mathrm{V}$ is the full depletion voltage. This relation shows that $\mathrm{N}_{\text {eff }} \alpha$ $\mathrm{VC}^{2}$, which may be simplified to $\mathrm{V} \alpha \mathrm{C}^{-2}$ for a constant effective carrier concentration, which is the case for uniform doping and is assumed for lifetime material. In any semiconductor, a rise in temperature will increase the current, since carriers become thermally activated to increase the effective carrier density, $\mathrm{N}_{\text {eff }}$, so that the current I $\alpha \mathrm{N}_{\text {eff }}$. An increase in light intensity is expected to have the same effect [9]. Because the current is ohmic and is generated in the whole of the depletion region, the depletion width becomes a function of depletion voltage. The capacitance becomes a function of radiation and temperature since electrons and holes are thermally activated.

\section{Modeling Description}

\section{A. Optical and electrical properties analysis}

The dark current, ID, for a device having depletion depth W, active area $A$ and the effective carrier concentration, $\mathrm{N}_{\text {eff }}$ under high temperature irradiation $T$ and gamma radiation fluence $Y$ is given by $[15,16]$ :

$I_{D}=\frac{q A W(T, \gamma) N_{e f f}(T, \gamma)}{2 \tau_{r}(T, \gamma)}$

Where $\mathrm{T}_{r}$ is the minority carrier lifetime after irradiation and it is given by $[9,12]$ :

$1 / \tau_{r}=1 / \tau_{0}+K_{r} \gamma$

Where $\mathrm{T}_{0}$ denotes the pre-irradiation minority carrier lifetime respectively, and $\mathrm{K}_{\mathrm{r}}$ is the damage coefficient for $\mathrm{T}_{\mathrm{r}}$. Assuming $\mathrm{a}$ linear relationship between damage increase and fluence, the damage coefficient for dark current $K_{D}$ and light photocurrent $\mathrm{K}_{\mathrm{p}}$, can be defined by following equation [15]:

$\Delta I_{D, P}=\left|I_{D, P}(\gamma)-I_{D, P}(0)\right|=K_{D, P} \gamma$

A simple model of the annealing can be constructed if we assume that the radiation-induced defects anneal according to a first-order mechanism (exponential recovery) [17], at a given absolute high temperature irradiation $\mathrm{T}, \mathrm{K}_{\mathrm{D}}$ can be related to an activation energy $E$ by the Arrhenius formula:

$K_{D}(T)=K_{D}(0) \exp \left(E / K_{B} T\right)$

Where $K_{B}$, is Boltzmann's constant.

Based on the data of [18-20], the following nonlinear thermal and radiation relations for the set Si PIN photodiode:

$I_{\text {Dark,Photo }}(T, \gamma)=I_{\text {Dark,Photo }}(T) \times I_{\text {Dark,Photo }}(\gamma)$

$\left.\begin{array}{c}I_{\text {Dark }}=I_{\text {Dark }}(0)+3.29 \times 10^{-24} \exp (831 / T) \times \gamma \\ I_{\text {Photo }}=I_{\text {Photo }}(0)-7.8 \times 10^{-14}\left(\begin{array}{c}-1.137 \times 10^{-11} T^{2} \\ +6.536 \times 10^{-9} T \\ +8.207 \times 10^{-8}\end{array}\right) \times \gamma\end{array}\right\}$

The drift current density of PIN photodiode is given as:

$$
j_{d r i f t}=q \int_{0}^{W} G_{o p t}(x) d x=q \varphi_{0}\left(1-e^{-\alpha W}\right)=\frac{q p_{0}\left(1-r_{f}\right)}{a h v}\left(1-e^{-\alpha W}\right)
$$

The one-dimension diffusion equation for initial minority holes $\mathrm{PnO}$ in the bulk $\mathrm{n}$ region is $[11,21]$

$$
\begin{aligned}
& D_{P} \frac{\partial^{2} p_{n}}{\partial x^{2}}-\frac{p_{n}-p_{n 0}}{\tau_{p}}+G_{\text {opt }}(x)=0 \\
& p_{n}{ }^{2}-\left(\frac{1}{L_{p}^{2}}\right) p_{n}=-\frac{p_{n} 0}{L_{p}^{2}}-\frac{\varphi_{0} \alpha}{D_{P}} e^{-\alpha x} \\
& D_{p}=\frac{L_{p}^{2}}{\tau_{p}}=\frac{K_{B} T}{q} \mu
\end{aligned}
$$

Where $T_{p}, L_{p}$ and $D_{p}$ are minority carrier life time, diffusion length and diffusion constant and $\mu$ is the carrier mobility. The solution of Eq. 11 under the boundary conditions $P_{n}=P_{n o}$ for $\mathrm{x}=\infty$, and $\mathrm{P}_{\mathrm{n}}=0$ for $\mathrm{x}=\mathrm{W}$.

$$
j_{\text {diffu }}=q p_{n 0} \frac{D_{p}}{L_{p}}+q \varphi_{0}\left(\frac{\alpha L_{p}}{1+\alpha L_{p}}\right) e^{-\alpha W}
$$


$j_{\text {total }}=j_{\text {drift }}+j_{\text {diffu }}$

$j_{\text {total }}=q \varphi_{0}\left(1+\frac{\alpha L_{p}-1-\alpha L_{p}}{1+\alpha L_{p}} e^{-\alpha W}\right)+q p_{n 0} \frac{D_{p}}{L_{p}}$

Under normal operating conditions, the dark-current term involving pno is much smaller so that the total photocurrent is proportional to the incident photon flux per unit area, $\varphi_{0}$. But under irradiation condition the total photocurrent is given by [9]:

$$
I_{\text {total }}=I_{\text {Photo }}+I_{\text {Dark }}
$$

Where Itotal is the current measured under illumination, IDark is the current measured in the dark, Iphoto is the current due to the illumination only. The high-temperature irradiation induce diffusion length change can be expressed as the following expression:

$I_{\text {photo }}=j_{\text {photo }} \times a=\frac{a q p_{0}\left(1-r_{f}\right)}{h v}\left(1-\frac{e^{-\alpha W}}{1+\alpha L_{p}}\right)$

$L_{p}=\left(\frac{1}{\alpha_{s}}\right)^{1-e^{\alpha W}\left(1-\frac{I_{\text {photo }}}{q \varphi_{0}}\right)} \frac{e^{\alpha W}\left(1-\frac{I_{\text {photo }}}{q \varphi_{0}}\right)}{e^{\alpha}}$

Where $a$ is the photodiode area. By comparing the different cases of the depletion layer width, the junction capacitance and the inverse of the absorption coefficient, a reasonable compromise between high-frequency response and high quantum efficiency of photodiode is found for absorption region thicknesses between $1 / \alpha$ and $2 / \alpha$.

$$
\alpha W=\frac{W}{(1 / \alpha)}=-\ln \left(\left(1+\alpha_{s} L_{p}\right)\left(1-\frac{I_{\text {photo }}}{a q \varphi_{0}}\right)\right)
$$

Irradiation induced change of the depletion layer width and the absorption coefficient must be take into consideration. Based on Eq.2 and the results of $[5,6]$ which shows the variation of the effective carrier concentration, Neff of Si PIN photodiode with electron irradiation dose. The depletion layer capacitance with its initial value $C_{0}$, when a voltage $V$ is applied to a junction with the built-in potential $\left(\mathrm{V}_{\mathrm{bi}}\left(\mathrm{S}_{\mathrm{i}}\right) \sim 0.65 \mathrm{v}\right.$ [9]), is given by:

$$
C(T, \gamma)=\frac{C_{0} \sqrt{\exp (-\beta \gamma)}}{a_{1}} \times \frac{\left(1+a_{2} T\right)}{\sqrt{|V|+V_{b i}}}
$$

Where $\mathrm{T}$ is the ambient temperature, $\mathrm{\gamma}$ is irradiation fluence, $\mathrm{C}_{\mathrm{d}}$ $a_{1}=1.176 \times 10-9, a 2=0.001052, . \beta=1.139 \times 10-15$ and $V=-1$ volt. The depletion width $\mathrm{W}$ can be expressed as the following [9] $W=\sqrt{\frac{2 \varepsilon\left(|V|+V_{b i}\right)}{q N_{e f f}}}=\sqrt{2 \varepsilon \rho \mu\left(|V|+V_{b i}\right)}$

$W=\frac{\varepsilon_{0} \varepsilon_{r} A}{C}$

Where $A$ is the effective photodiode area, $q$ is the electronic charge, Neff is the carrier concentration, $\varepsilon_{r}$ is the dielectric constant of silicon, $\varepsilon_{0}$ is the vacuum permittivity. From the previous results $[4,5]$, we can observed that $\varepsilon_{r}$ is constant for radiation but it is function of temperature as [22]:

$$
\begin{aligned}
\left.\varepsilon_{r}(T)\right|_{S i} & \approx 11.631+1.0268 \times 10^{-3} \mathrm{~T}+1.0384 \times 10^{-6} T^{2} \\
& -8.1347 \times 10^{-10} T^{3}
\end{aligned}
$$

The Tauc model [23] has been used as a stander model whereby the optical gap of an amorphous semiconductor may be determined as:

$\alpha(h v)=\alpha_{0}\left(h v-E_{g}\right)^{2}$

Where $1 / \alpha_{0}$ is the band edge parameter and $E_{g}$ is energy gap. The energy gap of the perfect silicon as a function of the temperature is given by [24]:

$E_{g}=1.166-\frac{4.731 \times 10^{-4} T^{2}}{636+T}$

But for imperfect semiconductor as a result of radiation induced defects the energy band gab $\mathrm{E}_{\mathrm{g}}$ is replace by $\mathrm{T}_{\text {auc }}$ bandgap energy $\mathrm{E}_{\mathrm{g}} \mathrm{T}_{\text {auc }}[23]$, then $\alpha$ will became:

$\alpha_{\text {Tauc }}(h v)=\alpha_{0}\left(h v-E_{\text {gTauc }}\right)^{2}$

In this case the residual absorption near the bandgap due to the intragap is called the $\mathrm{Ur}_{\text {bach }}$ tail [25], and can be expressed with the following equation close to the bandgap:

$\alpha_{U r b}(h v)=A_{0} \exp \left(\frac{\left(h v-E_{g}\right)}{E_{U r b}}\right)$

We need a function for $\alpha$ that is valid for the entire spectral range, i.e. an equation that combines (15) and (16) is smooth at the cross point, Ecross:

$\alpha_{\text {Tauc }}\left(E_{\text {cross }}\right)=\alpha_{U r b}\left(E_{\text {cross }}\right)$

$\alpha_{\text {Tauc }}^{\prime}\left(E_{\text {cross }}\right)=\alpha_{U r b}^{\prime}\left(E_{\text {cross }}\right)$

Where $\alpha^{\prime}$ denotes the first derivative with respect to the energy. With equations (28) and (29) the following conditions are obtained:

$E_{g_{\text {Tauc }}}=E_{g}-2 E_{U r b}\left(1+\mathrm{h}\left(\frac{1}{2 E_{U r b}} \sqrt{\frac{\alpha_{0}}{A_{0}}}\right)\right)$

$E_{U r b}(T)=E_{u 0} \times T$

Where Tauc and Urbach parameters of silicon material are $A_{0}=800 \mathrm{~cm}-1, E_{u 0}=36 \operatorname{Mev}$ [25], and $\alpha_{0}=4685 \mathrm{~cm}-1$ [26].

\section{B. Photodiode response analysis}

The responsitivity, S, of a PIN photodiode can be expressed as:

$$
S(T, \gamma)=\frac{I_{p h o t o}}{P_{0}}=\frac{q \eta}{h v}
$$

Where the quantum efficiency, $\eta$, can be given by:

$\eta(T, \gamma)=\frac{I_{\text {photo }} / q}{P_{0}(1-r) / h \nu}=\left(1-R_{f}\right)\left(1-\frac{e^{-\alpha W}}{1+\alpha L_{p}}\right)$

In order to analyze the response time of irradiated PIN photodiode, assume a modulated photon flux density as:

$\varphi=\varphi_{0} \exp (j \omega t)$ photons $\left(s . m^{2}\right)$

$\mathrm{T}_{\mathrm{o}}$ fall on photodiode, where $\omega$ is the sinusoidal modulation frequency. The total current through the depletion region generated by this photon flux can be shown to be $[21,27]$ :

$$
J_{\text {tctal }}=\left(\frac{j \omega \varepsilon\left(|V|+V_{b i}\right)}{W}+q \varphi_{0} \frac{1-e^{-j \omega t_{d r}}}{j \omega t_{d r}}\right) e^{j \omega t}
$$




$$
\left|\frac{I_{\text {total }}}{a q \varphi_{0}}\right|=\sqrt{\frac{\operatorname{Sin}^{2}\left(\frac{\omega t_{d r}}{2}\right)}{\left(\frac{\omega t_{d r}}{2}\right)^{2}}\left(1-\frac{\omega \varepsilon\left(|V|+V_{b i}\right)}{W\left(\omega t_{d r}\right)^{2}}\right)+\left(\frac{\omega \varepsilon\left(|V|+V_{b i}\right)}{W}\right)^{2}}
$$

Where $\varepsilon$ is the material permittivity, $t_{d r}$ is the transit drift time of carriers through the depletion region is:

$t_{d r}=W / 2 v_{d}$

For $\mathrm{p}$ on $\mathrm{n}$ devices where $\mathrm{W}$ is the width of the depletion region and ud is the average drift velocity of the carriers. In terms of measurable components Eq.37 The transit drift time becomes:

$$
t_{d r}=\frac{W^{2}}{\mu\left(|V|+V_{b i}\right)}
$$

Where $\mu$ is the carrier mobility.

The time for diffusion of carriers from the undepleted region to the depleted region is given by:

$t_{D f}=\frac{\ell^{2}}{2 D}$

Where $\mathrm{D}$ and $\ell$ are the diffusion constant and the undepleted thickness, which changes with the changing of the depletion layer width $\mathrm{W}$. The time constant $\mathrm{t}_{\mathrm{RC}}$ of the photodiode with a load resistance $R_{L}$ is given by:

$t_{R C}=2.2\left(R_{S}+R_{L}\right) C$

Where $\mathrm{C}$ is the capacitance of photodiode at applied bias $\mathrm{V}, \mathrm{R}_{\mathrm{s}}$ is the series resistance of photodiode, it is the resistance of the contacts and the undepleted bulk of the substrate.

$R_{S}=\frac{\rho\left(W_{0}-W\right)}{A}+$ contact resistore

Where $\mathrm{W}_{0}$ is the substrate thickness. Finally, for fully depleted photodiodes the rise time $t_{r}$ and fall time are generally the same.

$$
t_{r}=\sqrt{t_{d r}^{2}+t_{D f}^{2}+t_{R C}^{2}}
$$

Finally the power signal-to-noise ratio (SNR) S/N at the output of an analog optical receiver is defined by [11]:

$$
S / N=\frac{0.5 m^{2} I_{\text {photo }}^{2}}{\left(2 q f_{-d B}\left(I_{\text {photo }}+I_{\text {Dark }}\right)+4 K_{B} T f_{-d B} F_{n} / R_{L}\right)}
$$

Where $m$ is the analoge modulation index, $f_{-d \mathrm{~B}} \approx 0.35 / t_{r}$ is bandwidth and $t_{r}$ is rise time. The term $\left(4 \mathrm{~K}_{B} T f_{-d B} F_{n} / R_{L}\right)$ is the total noise associated with amplifier, it is referred to thermal noise of load resistor $R_{L}$ by the amplifier noise Fig. $F_{n}$.

\section{Results and Discussion}

Based on the above modeling equations analysis, the dynamic electro-optical characteristics of S. PIN photodiode are processed in high temperature gamma rays irradiations fields. The double impact of thermal and radiation effects are analyzed over ranges of causes (affecting parameters). As $1012>$ y, Fluence, e/cm2>1014 and 300>T, Temperature, $K>400$. Special software programs are designed, cast and employed to handle the given basic model, where variation of set of electrical and optical devices parameters $\{$ ID, IP, $\alpha, \eta\}$ against variations of a set of two effects $\{T, \gamma\}$ are processed. The device parameters are computed on bases of results of $[5$, $6,18,19,20]$. These variations will effect on response time tr, responsivity $\mathrm{S}$ and signal to noise ratio SNR of the device.
Based on the assumes set of the operating parameters, and the equations analysis, then the obtained results are displays in Fig. 1-7, for the processed Si device of optical wavelength of $\lambda=950 \mathrm{~nm}$ are assured the following facts:

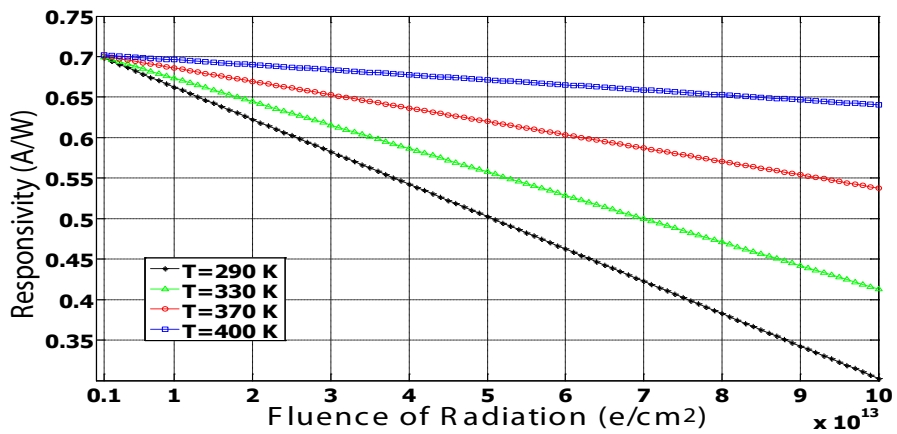

Fig. 1: Optical sensitivity of irradiated Si PIN photodiode at various radiation fluence and temperature.

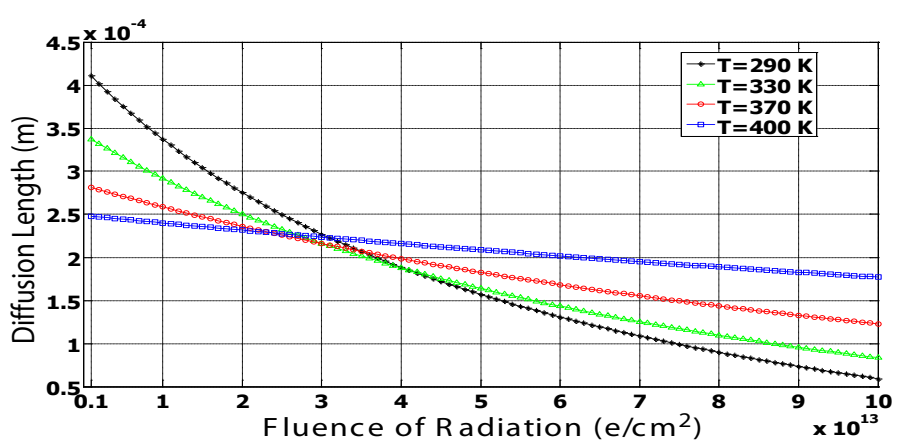

Fig. 2: Diffusion Length of irradiated Si PIN photodiode at various radiation fluence and temperature.

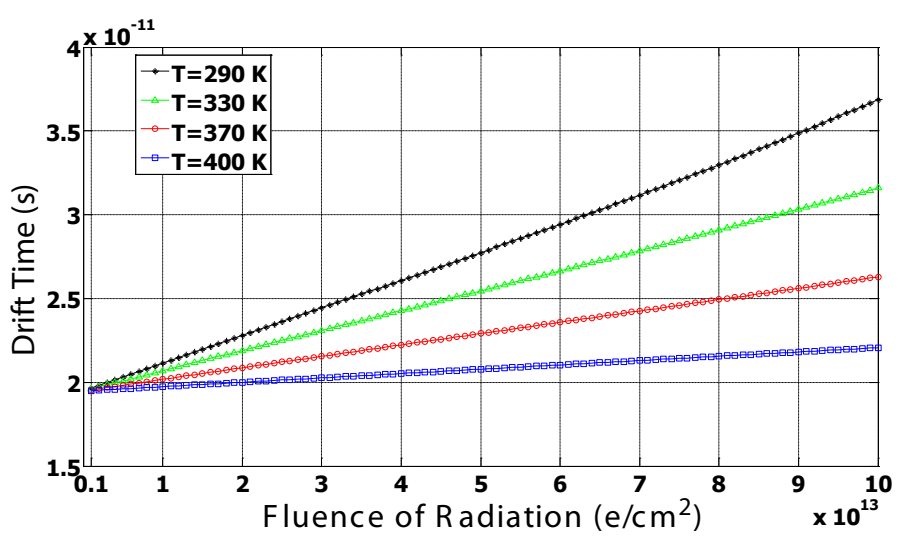

Fig. 3 : Drift Time of irradiated Si PIN photodiode at various radiation fluence and temperature.

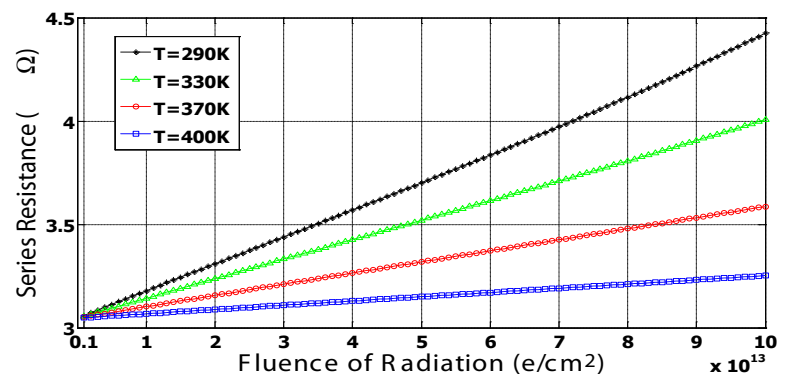

Fig. 4: Series Resistance of irradiated Si PIN photodiode at various radiation fluence and temperature. 


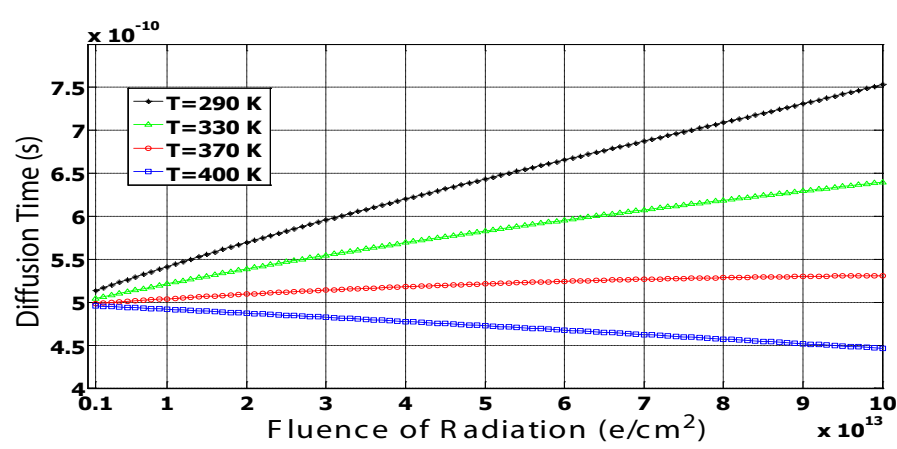

Fig. 5: Diffusion Time of irradiated Si PIN photodiode versus radiation fluence and temperature.

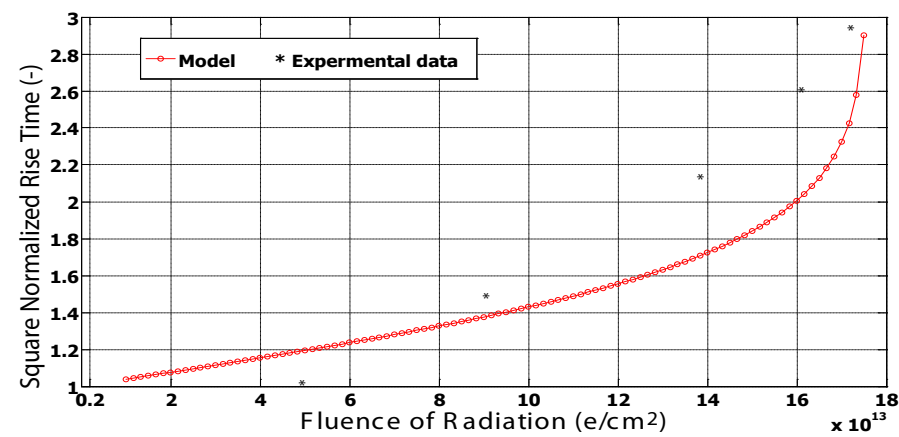

Fig. 6: Square rise time normalized to square rise time of unload Si PIN photodiode as function of radiation fluence.

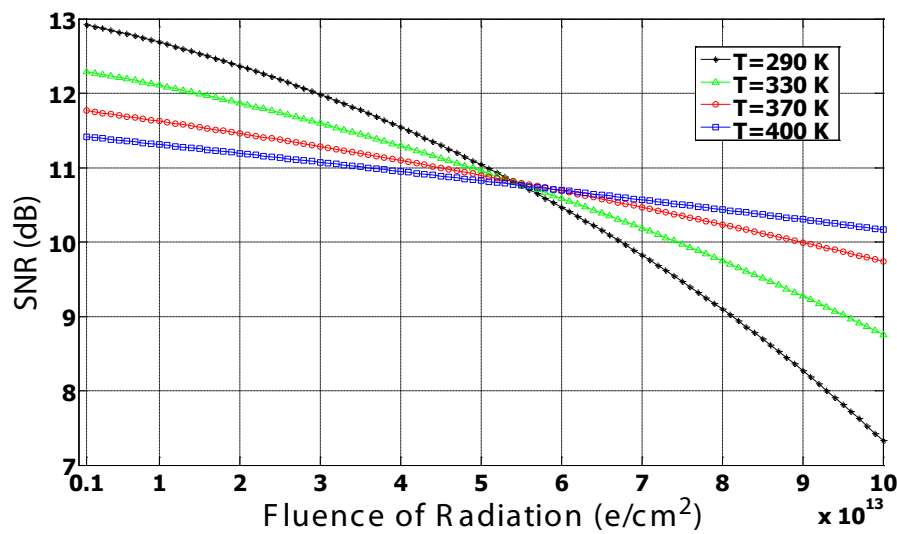

Fig. 7: Plot of SNR of irradiated Si PIN photodiode versus radiation fluence and temperature, $m=0.3, F_{n}=3 d B, R_{L}=50 \Omega$.

i) As shown in Fig. 1 has assured that as fluence of radiation on optoelectronic Si PIN device increases, this results in responsivity of device decreases at constant ambient temperature. But as ambient temperature increases, this leads to increase in responsivity of device at constant fluence of radiation.

ii) Fig. 2 has demonstrated that as fluence of radiation on optoelectronic Si PIN device increases, this results in diffusion length of carriers decreases at constant ambient temperature. Moreover as ambient temperature increases, this leads to increase in diffusion length of carriers at constant fluence of radiation.

iii) As shown in Fig. 3 has indicated that as fluence of radiation on optoelectronic Si PIN device increases, this results in drift time of carriers increases at constant ambient temperature. But as ambient temperature increases, this leads to decrease in drift time of carriers at constant fluence of radiation. iv) Fig. 4 has proved that as fluence of radiation on optoelectronic Si PIN device increases, this results in series resistance increases at constant ambient temperature. But as ambient temperature increases, this leads to decrease in series resistance at constant fluence of radiation.

v) As shown in Fig. 5 has indicated that as fluence of radiation on optoelectronic Si PIN device increases, this results in diffusion time of carriers slightly increases at constant ambient temperature. But as ambient temperature increases, this leads to decrease in diffusion time of carriers at constant fluence of radiation.

vi) Fig. 6 is confirmed that square of normalized rise time follow the empirical data [10] with the our model.

vii) Fig. 7 has assured that as fluence of radiation on optoelectronic Si PIN device increases, this results in signal to noise ratio decreases at constant ambient temperature. Moreover as ambient temperature increases, this leads to decrease in signal to noise ratio at constant fluence of radiation.

Therefore we can summarized the following results on the SI PIN photodiode performance in the following points:

a) The damage caused by fluence $y$ results in decreasing responsivity and signal to noise ratio whatever the set of effect ambient temperature T. But the annealing caused by ambient temperature $\mathrm{T}$, decrease slightly the negative effects of irradiation on optoelectronic device performance.

b) There is a delay in speed of the response as a result of increasing of the drift and the diffusion times with the increase of the irradiation fluence.

c) There is an increase in the series resistance Rs of the device with the irradiation fluence.

d) Radiation defect centers will reduce the minority carrier diffusion length in undepleted region, then the photocurrent will reduce.

\section{Conclusions}

In a summary, we have presented analytical modeling of the dynamic characteristics of Si PIN photodiode under high temperature gamma radiation. The modeling basics yields an analytical expression for the responsivity of optoelectronic device as a function of irradiation Fluence and temperature. It is evident that also enable better prediction of photocurrent levels, delays and signal bandwidth. Moreover we have demonstrated the circuit effects on signal performance that included as a value of signal to noise ratio. The degradation and delays can be explained by a decrease in the life time and diffusion length of minority carrier caused by the formation of radiation defects. It is found that the degradation of device performance decrease with increasing irradiation temperature. This result suggests that creation and recovery of the radiation damage proceeds simultaneously at high temperature degrees.

\section{References}

[1] Yu. K. Akimov, "Silicon Radiation Detectors: Instruments and Experimental Techniques," IEEE Trans. on Nucl. Sci., Vol. 50, No. 1, pp. 1-28, 2007.

[2] J.R. Srour, C. J. Marshall, P.W. Marshall, "Review of Displacement Damage Effects in Silicon Devices," IEEE Trans. on Nucl. Sci., Vol. 50, No. 3, pp. 653-670, 2003.

[3] A.M. Saad, "Effect of cobalt 60 and $1 \mathrm{MeV}$ electron irradiation on silicon photodiodes/solar cells," J. Phys. Vol. 80, pp.1591-1599, 2002. 
[4] M. Pattabi, S. Krishnan, G. Sanjeev, "Studies on the temperature dependence of $\mathrm{I}-\mathrm{V}$ and $\mathrm{C}-\mathrm{V}$ characteristics of electron irradiated silicon photo-detectors," J. Solar Energy Materials \& Solar Cells 91, pp.1521-1524, 2007.

[5] S. Krishnan, G. Sanjeev, M. Pattabi, " $8 \mathrm{MeV}$ electron irradiation effects in silicon photo-detectors," Nucl. Instr. and Meth. in Physics Research B 264, pp. 79-82, 2007.

[6] A. Simon, G. Kalinka, M. Jaks, Z. Pastuovic, M. Nova, A.Z. Kiss, "Investigation of radiation damage in a Si PIN photodiode for particle detector," Nucl. Instr. and Meth. in Physics Research B 260, pp. 304-308, 2007.

[7] M. Van Uffelen, Ivan Genchev, F. Berghmans, "Reliability study of photodiodes for their potential use in future fusion reactor environments," SPIE Proceeding 5465, pp. 92 102, 2004.

[8] K. Gill, S. Dris, R. Grabit, R. Macias, E. Noah, J. Troska, F. Vasey, "Radiation hardness assurance and reliability testing of InGaAs photodiodes for optical control links in the CMS experiment," IEEE Trans. On Nucl. Sci., Vol. 5 , No. 2, pp. 1480-1487, 2005.

[9] M. McPherson, "Infrared photoconduction in radiationdamaged silicon diodes," J. Opt. A: Pure Appl. Opt. 7, pp. S325-S330, 2005.

[10] Shinobu Onoda, Toshio Hirao, Hisayoshi Itoh, Tsuyoshi Okamoto, "Evaluation of Transient Current in Si PIN photodiode Induced by High-Energy Charged Particles," Proc. Sch. Eng. Sch. Inf. Sci. Tokai Univ. 31, pp. 1-4, 2006.

[11] G. Keiser, "Optical Fiber Communications", 4th Ed., Tata McGraw-Hill, New Delhi, 2008.

[12] M.A. Cappelletti, U. Urcola, E.L. Peltzer y Blanc`a., "Radiation-damaged simulation PIN photodiodes," Semicond. Sci. Technol., Vol. 21, pp. 346-351, 2006.

[13] S. Onoda, T. Hirao, J.S. Laird, H. Mori, T. Okamoto, Y. Koizumi, H. Itoh, "Spectral Response of a Gamma and Electron Irradiated Pin Photodiode," IEEE Trans. on Nucl. Sci., Vol. 49, No. 3, pp. 1446-1449, 2002.

[14] B. Danilchenko, A. Budnyk, L. Shpinar, D. Poplavskyy, S.E. Zelensky, K.W.J. Barnham, N.J. Ekins, “1 MeV electron irradiation influence on GaAs solar cell performance," J. Solar Energy Materials and Solar Cells 92, pp. 13361340, 2008.

[15] H. Ohyama, K. Takakura, K. Hayama, S. Kuboyama, Y. Deguchi, S. Matsuda, E. Simoen and C. Claeys, “Damage coefficient in high-temperature particle and $y$-irradiated silicon $\mathrm{p}-\mathrm{i}-\mathrm{n}$ diodes," J. Appl. Phys. Lett., Vol. 82, No. 2, pp. 296-298, 2003.

[16] M. A. Cappelletti, A.P. C'edola, E.L. Peltzer y. Blanc`a, "Simulation of silicon PIN photodiodes for use in spaceradiation environments," Semicond. Sci. Technol., Vol. 23, No. 2, pp. 7-13, 2008.

[17] J.R. Srour, Fellow, D.H. Lo, “Universal Damage Factor for Radiation-Induced Dark Current in Silicon Devices," IEEE Trans. on Nucl. Sci., Vol. 47, No. 6, pp. 2451-2459, 2000.

[18] K. Takakura, K. Hayama, D. Watanabe, H. Ohyama, T. Kudou, K. Shigaki, S. Matsuda, S. Kuboyama, T. Kishikawa, J. Uemura, E. Simoen, C. Claeys, "Radiation defects and degradation of Si photodiodes irradiated by neutrons at low temperature," Physica B 376-377, pp. 403-406, 2006.
[19] H. Ohyama, T. Hirao, E. Simoen, C. Claeys, S. Onoda, Y. Takami, H. Itoh, "Impact of lattice defects on the performance degradation of Si photodiodes by hightemperature gamma and electron Irradiation," Physica B 308-310, pp. 1226-1229, 2001.

[20] H. Ohyama, E. Simoen, C. Claeys, K. Takakura, H. Matsuoka, T. Jono, J. Uemura, T. Kishikawa, "Radiation damage in Si photodiodes by high-temperature Irradiation," Physica E 16, pp. 533 - 538, 2003.

[21] S. M. Sze, "Physics of Semiconductor Devices", John Wiley \& Sons, 3rd Ed, USA, 2007.

[22] H. Li, "Refractive Index of Silicon and Germanium and Its Wavelength and Temperature Derivatives, Journal of Physical and Chemical Reference Data," Vol. 9, No. 3, pp. 561-658, 1980.

[23] J. Tauc, "Amorphous and liquid semiconductors", New York, Plenum Press, 1974.

[24] Benjawan Kjornrattanawanich, Raj Korde, Craig N. Boyer, Glenn E. Holland, John F. Seely, "Temperature Dependence of the EUV Responsivity of Silicon Photodiode Detectors," IEEE Trans. on Electron Devices, Vol. 53, No. 2, pp.218 223, 2006.

[25] Y. Pan, F. Inam, M. Zhang, D. A. Drabold, "Atomistic Origin of Urbach Tails in Amorphous Silicon," J. Physical Review Letters, Vol. 20, No. 6, pp. 1-4, 2008.

[26] Martin A. Green, "Self-consistent optical parameters of intrinsic silicon at $300 \mathrm{~K}$ including temperature coefficients," J. Solar Energy Materials \& Solar Cells 92, pp. 1305- 1310, 2008.

[27] K. Konno, O. Matsushima, D. Navarro, M. Miura-Mattausch, "High frequency response of $p$-i-n photodiodes analyzed by an analytical model in Fourier space," J. Appl. Phys., Vol. 96, No. 7, pp. 3839-3844, 2004.

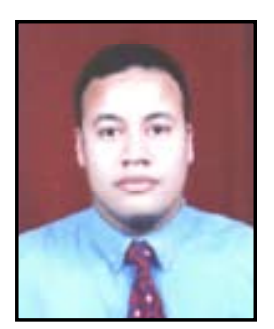

Dr. Ahmed Nabih Zaki Rashed Was born in Menouf city, Menoufia State, Egypt country in 23 July, 1976. Received the B.Sc., M.Sc., and Ph.D. scientific degrees in the Electronics and Electrical Communications Engineering Department from Faculty of Electronic Engineering, Menoufia University in 1999, 2005, and 2010 respectively. Currently, his job carrier is a lecturer in Electronics and Electrical communications Engineering Department, Faculty of Electronic Engineering, Egypt. His interesting research mainly focuses on transmission capacity, a data rate product and long transmission distances of passive and active optical communication networks. His areas of interest and experience in Optical communication systems, Advanced optical communication networks, Wireless optical access networks, Analog communication systems, Optical filters and Sensors, digital communication systems, Optoelectronics devices, and Advanced material science, Network management systems, Multimedia data base, Network Security, Encryption and optical access computing systems. He is a reviewer member in high quality scientific research internternational journals in the field of Electronics, Electrical communication and andvanced optical communication systems and networks. 